\title{
A Motor Driven by Permanent Magnets Alone; A Clean and Abundant Source of Electromagnetic Energy from Iron and Other Ferromagnetic Materials
}

\section{Kenneth Kozeka}

Austin Peay State University, Clarksville, USA

Correspondence to: Kenneth Kozeka, Kkozeka@kedronenergy.com

Keywords: Electromagnetic Energy, Permanent Magnets, Angular Momentum, Permanent Magnet Motor

Received: May 13, $2017 \quad$ Accepted: September 18, 2017

Published: September 21, 2017

Copyright $\odot 2017$ by authors and Scientific Research Publishing Inc.

This work is licensed under the Creative Commons Attribution International License (CC BY 4.0).

http://creativecommons.org/licenses/by/4.0/

\section{c) (i) Open Access}

\section{ABSTRACT}

Since the discovery of loadstone more than three hundred years ago, we have contemplated using the magnetic forces generated by permanent magnets to work for us. It is shown here that two permanent magnets with opposite poles facing can attract along their equatorial plane and then repel along their polar plane in sequence, without reversing polarity and without the use of another source of energy. This sequence of attract and repel between two permanent magnets is like the attract and repel sequence between an electromagnet and permanent magnet in an electric motor. The discovery described here can be used to construct a motor driven entirely by permanent magnets.

\section{INTRODUCTION}

Electromagnetism is one of the four fundamental forces that act on matter and it is second in effective strength. The relationship between moving electrons and magnetic fields is exploited today in a wide variety of applications and technologies that includes the use of electromagnets and permanent magnets. The quantum "spin" or angular momentum of electrons is believed to be responsible for generating the magnetic field in permanent magnets [1]. Most particles with spin possess a magnetic moment [2]. Richard Feynman (Nobel laureate, 1965) described iron as containing "a perpetually circulating current" from the spin of electrons [3]. The magnetic field is delivered or carried by a stream of "virtual" photons [4]. The source of energy responsible for the electron's spin and the momentum of photons is not known. It has been speculated that the energy may come from a loss of mass, dark energy, cosmic radiation or perhaps from another very small dimension. The perturbation theory proposes that a charged particle, the electron, can pass through an intermediate "virtual state" and emit a photon without violating energy conservation. How attracting and repelling forces are exerted between magnetic fields is also not known. Vortices and corkscrew interaction between the photon streams that comprise the magnetic fields has been proposed [5]. The continuous streams of virtual photons that flow from atomic electrons in ferromagnetic material 
are a source of electromagnetic force that can be converted into mechanical energy which can then be used to drive an electric generator. This abundant and clean source of energy has not been fully exploited and is receiving little attention at a time when alternatives to fossil fuels are direly needed.

In the early eighteenth century, long after the discovery of lodestone, the first compound permanent magnet was manufactured and available for commercial use [6]. Recent technological advances in the magnetization of magnetic materials produce extremely powerful and stable permanent magnets. Rare earth magnets containing lanthanide elements such as neodymium and samarium carry large magnetic moments. For example, a "neodymium" (NdFeB) magnet comprised of neodymium, iron and boron and measuring only $10.16 \mathrm{~m} \times 10.16 \mathrm{~cm} \times 5.08 \mathrm{~cm}$ can have a Brmax of 14,800, a surface field Gauss of 4,933, a pull force of $557 \mathrm{~kg}$, and is stable at $176^{\circ} \mathrm{F}$. Unless overheated or physically damaged, the magnet will lose less than $1 \%$ of its strength over 10 years.

\section{TYPICAL ELECTRIC MOTORS THAT USE PERMANENT MAGNETS AND ELECTROMAGNETS}

Powerful permanent magnets are used today in a wide variety of applications that include electric motors in which magnetic fields are used to convert electrical energy into mechanical energy. An electric motor that uses permanent magnets does not have field windings that serve as electromagnets on the stator frame. Instead, the permanent magnets on the stator frame provide the magnetic fields which interact with the rotor field to produce torque (Figure 1). This eliminates the need to power the stator thereby reducing electrical energy consumption. When electricity from an external source passes through the rotor field, it serves as an electromagnet that is attracted to the permanent magnet causing the motor to rotate (Figure 1(a)). For continued rotation, the electromagnet allows the rotor field to reverse the polarity of its magnetic field (Figure 1(b)) producing repulsion. The repulsive force between the poles repels the electromagnet along its path of motion. If polarity of the rotor is not reversed, the attractive force that pulled the electromagnet toward the permanent magnet will prevent the electromagnet from escaping and cause it to return and rest opposite the permanent magnet.

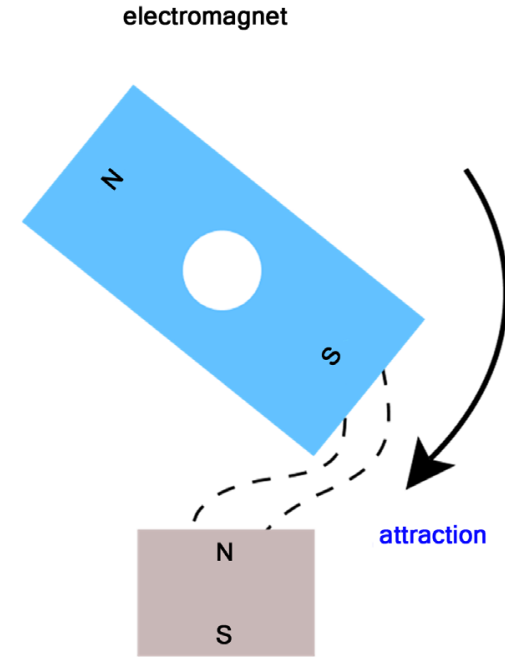

permanentmagnet

(a)

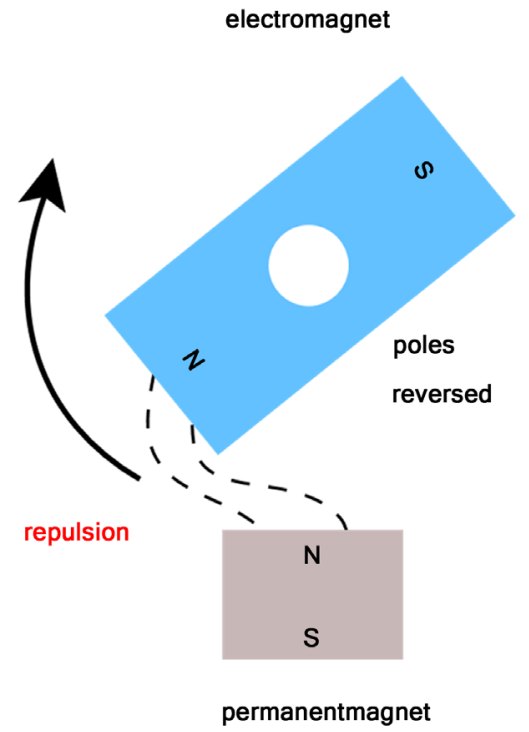

(b)

Figure 1. (a) The basic principle of an electric motor is illustrated with an electromagnet and a permanent magnet; (b) Continued motion beyond attraction occurs by reversing the polarity of the electromagnet to generate a repulsive force. 


\section{THE DISCOVERY OF SIMULTANEOUS RESULTANT ATTRACTION AND REPULSION BETWEEN TWO PERMANENT MAGNETS WITH OPPOSITE POLES FACING}

Electric motors, with or without the use of permanent magnets, produce rotation from a repeated sequence of attraction followed by repulsion which requires reversing polarity. For centuries, many unsuccessful attempts have been made to construct a motor using permanent magnets to generate the magnetic fields for both the stator and the rotor. Such a motor would be powered entirely by the intrinsic magnetic fields generated by permanent magnets. The discovery presented here allows permanent magnets to attract and repel in sequence producing continued motion like an electric motor and without reversing polarity or the use of an external source of energy.

Most of us have handled permanent magnets and experienced the attractive and repulsive forces that occur between them. It is easy to imagine having the magnets do work for us. For example, the attractive force between two sufficiently strong permanent magnets can move an object as the magnets pull themselves together. However, to have the magnets repeat this work, they must be pulled apart. The amount of work or mechanical energy required to pull the magnets apart is similar to the amount of mechanical energy the magnets generated when they pulled themselves together. Accordingly, permanent magnets are unable to work continuously on their own without an external source of mechanical energy to repeatedly pull them apart.

Figure 2 illustrates typical field lines between opposite poles of two permanent magnets that generate the commonly experienced attractive force exerted in the polar (vertical) plane. The attractive force generated by sufficiently strong magnets can pull the magnets together over a distance until they come to rest in contact with one another. The attractive force between opposite poles can also pull the magnets together along an equatorial (horizontal) plane until they come to rest opposite one another. Figure 3 illustrates the field lines responsible for this horizontal attraction.

It is common for attract and repel forces to exist between two magnets with opposite poles facing as illustrated in Figure 4. However, the repulsive force in the polar plane is usually very weak compared to the attractive force in the same plane causing the resultant force to be attraction. A unique condition was created in which the fields of two permanent magnets with opposite poles facing, generate a resultant attraction force between them in the equatorial plane and a resultant repulsion force in the polar plane, simultaneously. Figure 5 illustrates the field lines responsible for the simultaneous equatorial attract (blue arrows) and polar repel (red arrows) forces occurring between a pair of permanent magnets. The unusual resultant repulsion in the polar plane is a result of the shape of the magnets and their positions and is generated between like poles even though the magnets have their opposite poles facing one another.

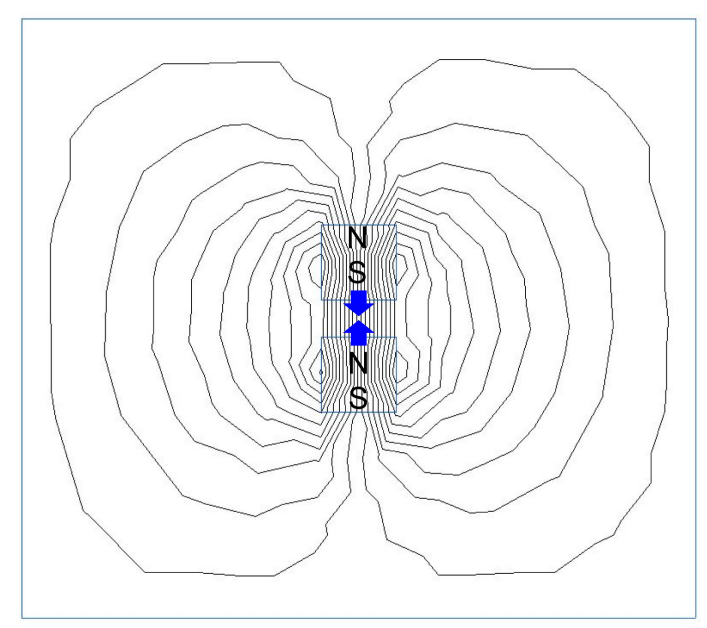

Figure 2. Polar attraction. Typical field lines that generate attractive forces (arrows) in the polar plane between opposite poles of permanent magnets. 


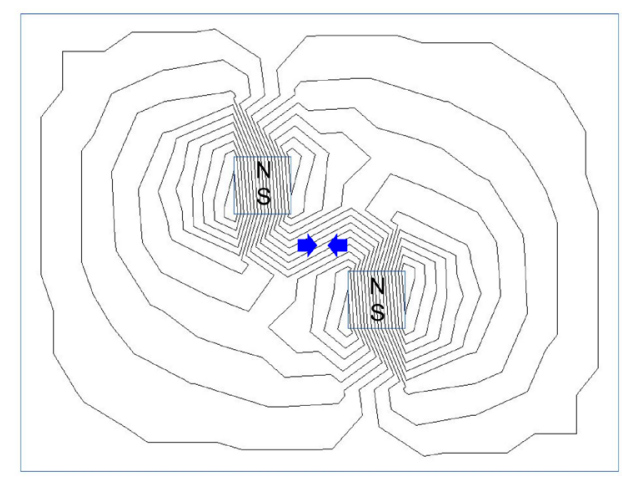

Figure 3. Equatorial attraction. Typical field lines that generate attractive forces (arrows) in the horizontal (equatorial) plane between opposite poles of permanent magnets.

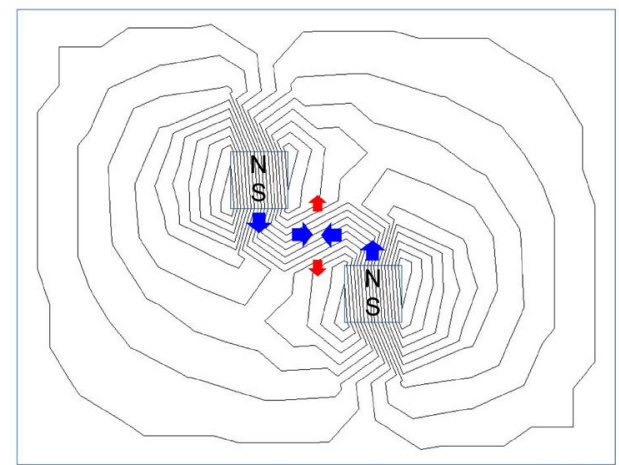

Figure 4. Resultant attraction (blue arrows) in the polar and equatorial planes between two magnets with opposite poles facing. Weak repulsion (red arrows) in the polar plane commonly exists between permanent magnets with opposite poles facing.

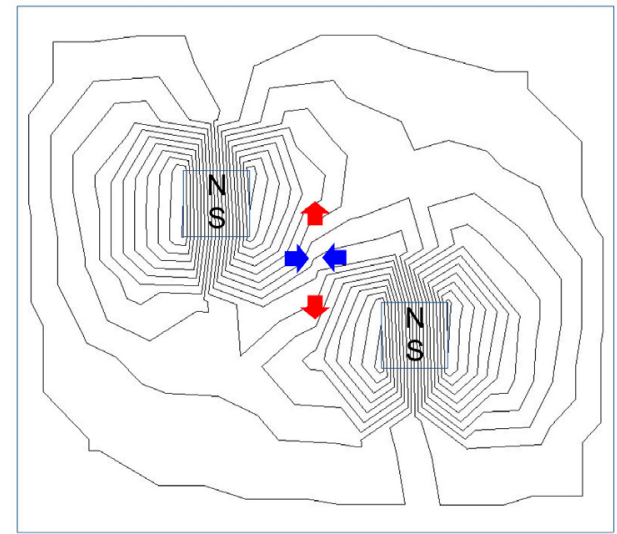

Figure 5. Simultaneous resultant equatorial attraction and polar repulsion between magnets with opposite poles facing. Blue arrows indicate the field lines responsible for the equatorial attraction and red arrows indicate the field lines responsible for the polar repulsion. 


\section{UNAIDED SEQUENCE OF ATTRACT AND REPEL BETWEEN PERMANENT MAGNETS}

The resultant attractive force exerted in the equatorial plane can be used to pull the magnets together horizontally. The resultant repulsive force exerted in the polar plane can, in turn, be used to push the magnets apart vertically without reversing polarity or the use of other energy. Thus, two permanent magnets are made to pull themselves together with an attractive force and then push themselves apart unaided by an external force or other energy. Normally, an external force must be applied to separate two permanent magnets that have pulled themselves together with their attractive force. Until now, we have not observed two permanent magnets attract and repel themselves in sequence, unaided. This sequence of attraction followed by repulsion is like the attract-repel sequence that occurs in an electric motor between a permanent magnet and an electromagnet.

\section{A DEMONSTRATION OF THE ATTRACT-REPEL SEQUENCE}

An apparatus was constructed to demonstrate the simultaneous resultant attraction in the equatorial plane and the resultant repulsion in the polar plane between two permanent magnets with opposite poles facing one another. To reduce friction during motion, the permanent magnets were attached to carts with 8 wheels (Figure 6). Four permanent magnets were positioned on each cart as shown in Figure 6 and illustrated in Figure 7. The magnets were made of neodymium (NdFeB), grade 52, and measured $5.08 \mathrm{~cm}$ long, $2.54 \mathrm{~cm}$ wide and $1.27 \mathrm{~cm}$ thick. They were magnetized through their $1.27 \mathrm{~cm}$ thickness. Each magnet has a Brmax of 14,800 Gauss and a pull force of $41.28 \mathrm{~kg}$. Rails were constructed to limit motion of the carts to vertical and horizontal paths as shown in Figure 8. To minimize magnetic interference, the carts and rails were made of aluminum alloy and the screws and nuts were made of brass.

In the apparatus (Figure 8) and illustration (Figure 9), horizontal motion of magnet $\mathrm{H}$ begins with magnets $\mathrm{H}$ and $\mathrm{V}$ separated by $8.89 \mathrm{~cm}$ in the equatorial (horizontal) plane and $3.81 \mathrm{~cm}$ in the polar (vertical) plane. Magnet $\mathrm{V}$ is stationary (Figure 9(a)). A resultant attractive force between the magnets in the equatorial (horizontal) plane pulls magnet $\mathrm{H}$ toward magnet $\mathrm{V}$ (Figure 9(b), Movie S1). Magnet $\mathrm{H}$ is forced to stop $5.08 \mathrm{~cm}$ away from magnet $\mathrm{V}$ (Figure 7(b)). In this position, a resultant repulsive force exists between the magnets in the polar (vertical) plane. With magnet $\mathrm{H}$ now stationary, magnet $\mathrm{V}$ is released. The repulsive polar force pushes magnet V vertically away from magnet $H$ (Figure 7(c), Movie S2). Magnet V travels $3.81 \mathrm{~cm}$ before striking the end of the vertical rail (Figure 7(c)). If allowed, magnet $\mathrm{V}$ would travel further vertically. Movie S3 demonstrates the horizontal and vertical movements in sequence.

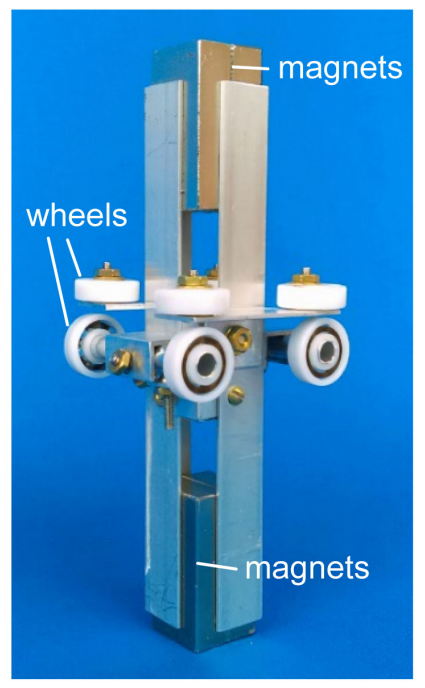

Figure 6. A perspective view of the cart with the permanent magnets held in place above and below. Glass ball bearings in acetal races serve as wheels. 


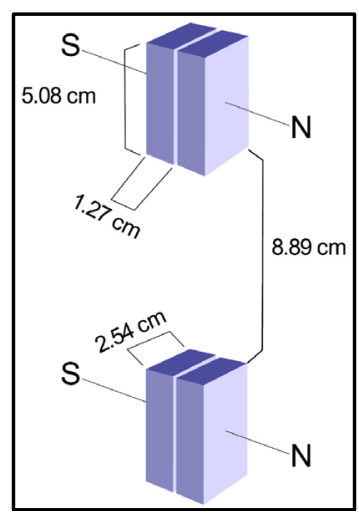

Figure 7. A perspective view of the four permanent magnets on each cart. The magnets were magnetized through their thinness.

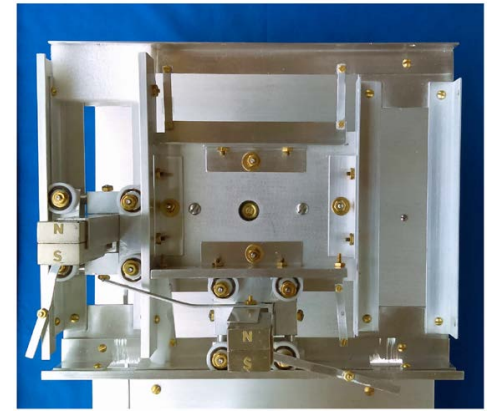

Figure 8. A superior view of an apparatus that demonstrates horizontal attraction followed by vertical repulsion of permanent magnets. The magnets in the image are simultaneously exerting a resultant attraction in the polar (horizontal) plane and a resultant repulsion force in the polar (vertical) plane.

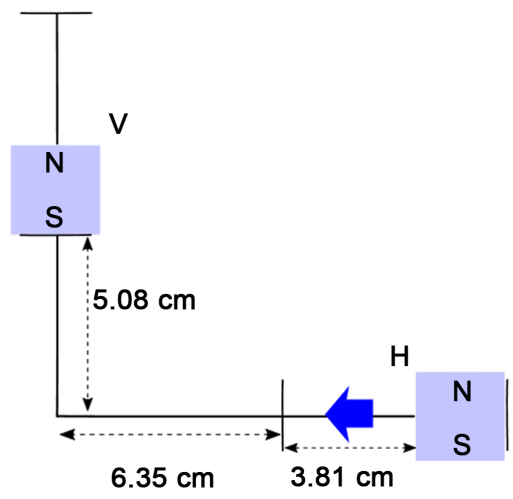

(a)

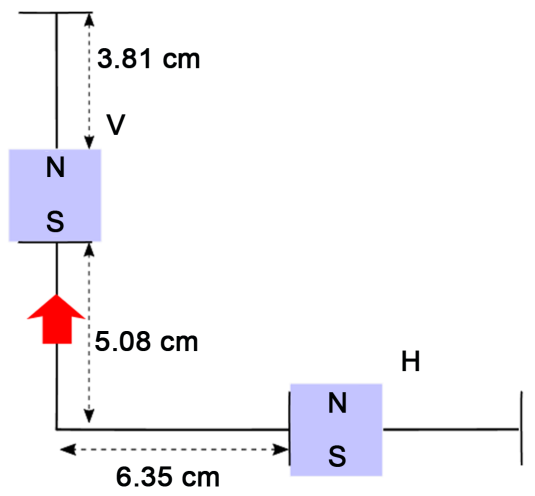

(b)

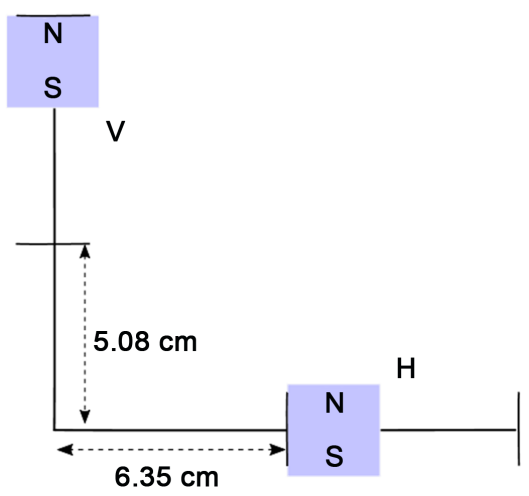

(c)

Figure 9. The positions and distances traveled by the permanent magnets in the apparatus. (a) Equatorial attraction. A resultant attraction in the equatorial plane acts on permanent magnet $\mathrm{H}$ to move it horizontally toward permanent magnet V. This is demonstrated in Movie S1; (b) Polar repulsion. A resultant repulsion in the polar plane acts on permanent magnet $\mathrm{V}$ to move it vertically while magnet $\mathrm{H}$ is at rest. This is demonstrated in Movie S2; (c) Magnet V is at rest after being repelled vertically. The complete attract-repel sequence is demonstrated in Movie S3. 


\section{MEASUREMENTS OF THE ATTRACTIVE AND REPULSIVE FORCES}

The resultant attractive and repulsive forces were measured using an IMADA model DS2-110 digital force gauge. Resultant forces applied to the carts were measured at $3.18 \mathrm{~mm}$ intervals along the horizontal and vertical paths of motion as described in Figure 9. Measurements of the resultant attractive force in the equatorial plane responsible for horizontal motion are shown in Figure 10. Measurements of the resultant repulsive force in the polar plane responsible for vertical motion are shown in Figure 11. The combined force values from the attractive force in the equatorial plane and repulsive force in the polar plane is presented in Figure 12. A surplus of mechanical energy is available from total force (mechanical energy)

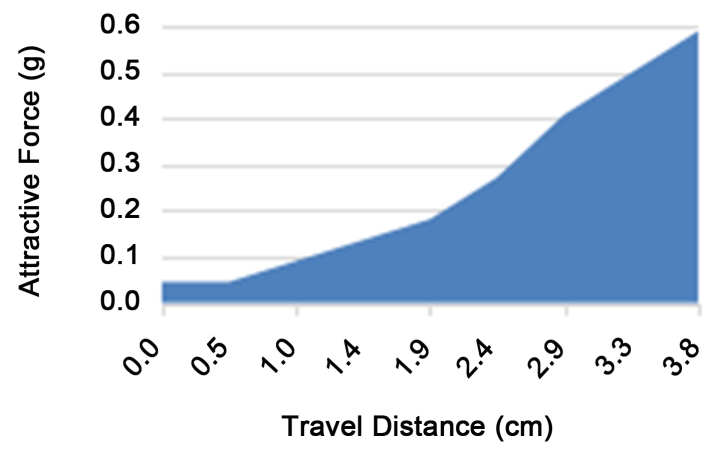

Figure 10. Equatorial attractive force versus distance. Force measurements were taken at $3.18 \mathrm{~mm}$ intervals over the distance traveled in the equatorial plane.

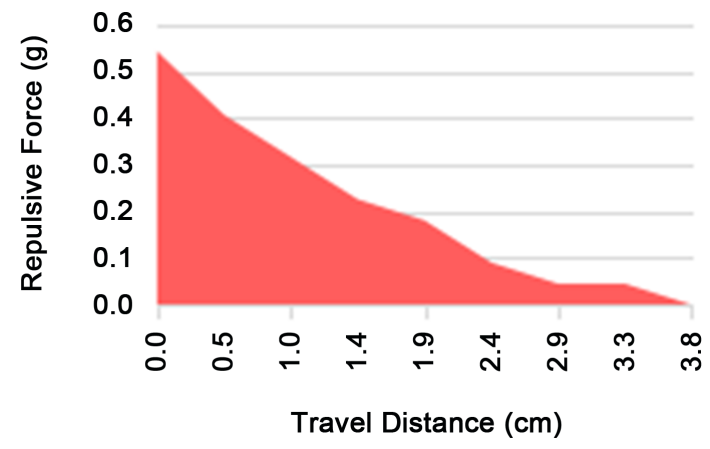

Figure 11. Polar repulsive force versus distance. Force measurements were taken at $3.18 \mathrm{~mm}$ intervals over the distance traveled in the polar plane.

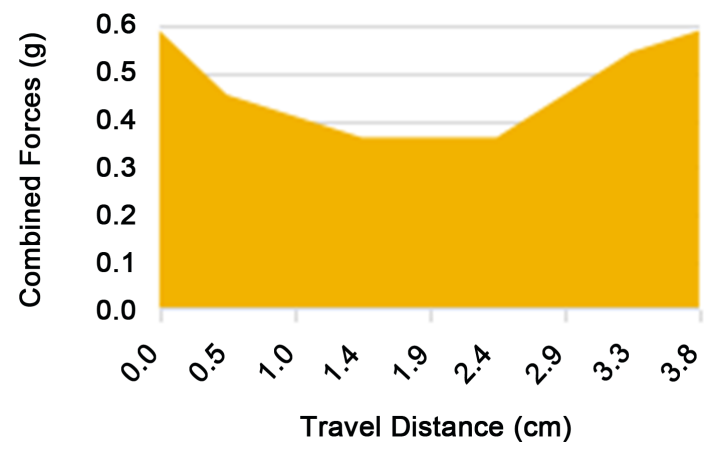

Figure 12. Combined attractive and repulsive forces shown in Figure 10 and Figure 11. 
available during the attraction and repulsion phases. This surplus of energy can be used to do work such as drive an electric generator. The data provided here are intended only to illustrate the phenomenon and do not represent the optimal conditions for maximum energy output.

\section{MOTOR DESIGN CONSIDERATIONS}

Repeating the sequence of attraction and repulsion described here requires that the magnets return to their starting positions. However, the short distance traveled by the magnets described here leaves them within the attractive and repulsive fields responsible for their initial motion. Therefore, returning the magnets to their starting positions would be opposed by these residual attractive and repulsive forces respectively. A considerable amount of the mechanical energy would have to be spent to overcome these forces when returning the magnets. These opposing residual forces and the expenditure of energy to overcome them can be greatly reduced by extending the distance traveled by magnets $\mathrm{H}$ and $\mathrm{V}$. For example (Figure 13), if magnet $\mathrm{V}$ were made to travel $15.24 \mathrm{~cm}$ vertically instead of $6.35 \mathrm{~cm}$, magnet $\mathrm{H}$ could then move back horizontally to its starting position without encountering significant opposition from the attractive force in the polar plane. With extended travel distances, the resultant attractive force in the equatorial plane initially will be too weak to pull magnet $\mathrm{H}$ horizontally. Figure 13 illustrates how pairs of magnets can be linked together to tow one another along a portion of their travel. The repulsive force between magnets $\mathrm{V} 1$ and $\mathrm{H} 1$ is sufficiently strong for magnet $\mathrm{V} 1$ to tow magnet $\mathrm{H} 2$ in closer to magnet $\mathrm{V} 2$ where the attractive forces are stronger. In turn, the attractive force between magnets $\mathrm{V} 2$ and $\mathrm{H} 2$ can tow magnet V1 further away from magnet H1. Movie S4 demonstrates motion generated by two pairs of magnets linked together as illustrated in Figure 13. This connection of magnet pairs in different phases of the attraction and repulsion sequence is like the arrangement of pistons in a combustion engine in which the combustion stroke of one piston drives the exhaust stroke of another piston. Another similarity exists in that both magnets and pistons travel along linear paths as they provide mechanical energy.

Mechanical energy can be conserved by making the magnets work at both ends of their travel. In this manner, energy is not wasted returning the magnets to their original positions to repeat the cycle. Connecting four pairs of magnets completes the cycle allowing for continued motion driven entirely by permanent magnets. An animation demonstrating this sequence of motion is shown in Movie S5.

It is noted here that the stator and rotor in a conventional electric motor requires several pairs of magnets to achieve continued motion. The attraction and repulsion sequence between one stator magnet and the electromagnet (rotor) cannot produce sufficient inertia to turn the rotor one full rotation and repeat the cycle. Likewise, the method described here requires the use of multiple pairs of permanent magnets to extend travel distances and complete a cycle.

\section{COMMERCIAL APPLICATION}

Our present understanding of electrons, virtual photons, electromagnetic energy and magnetic force supports the possibility of a motor driven entirely by permanent magnets. The method described here illustrates how permanent magnets alone can be used to produce continuous motion and provide a surplus of mechanical energy that can be used for other purposes such as driving an electric generator.

For many reasons, electromagnetic energy from permanent magnets is a highly practical, clean, and abundant source of energy. Electromagnetic force has been calculated to be 39 orders of magnitude stronger than gravitational force and its intrinsic source is abundant. The amount of energy required to create permanent magnets is insignificant compared to the amount of electromagnetic energy intrinsically available from them after they are magnetized. Iron, the most common ferromagnetic substance, is the second most abundant metal on Earth. The powerful magnets containing neodymium and samarium are not required to generate a practical amount of net mechanical energy using the method described here. Other weaker permanent magnets can be used. The stability (coercivity) and strength (magnetic moment) of permanent magnets today are extremely high. The magnitude of the electromagnetic forces exerted between magnet pairs to generate mechanical energy as described here is below the coercivity value of the magnets. Therefore, the magnets will remain stable under normal operating conditions. 


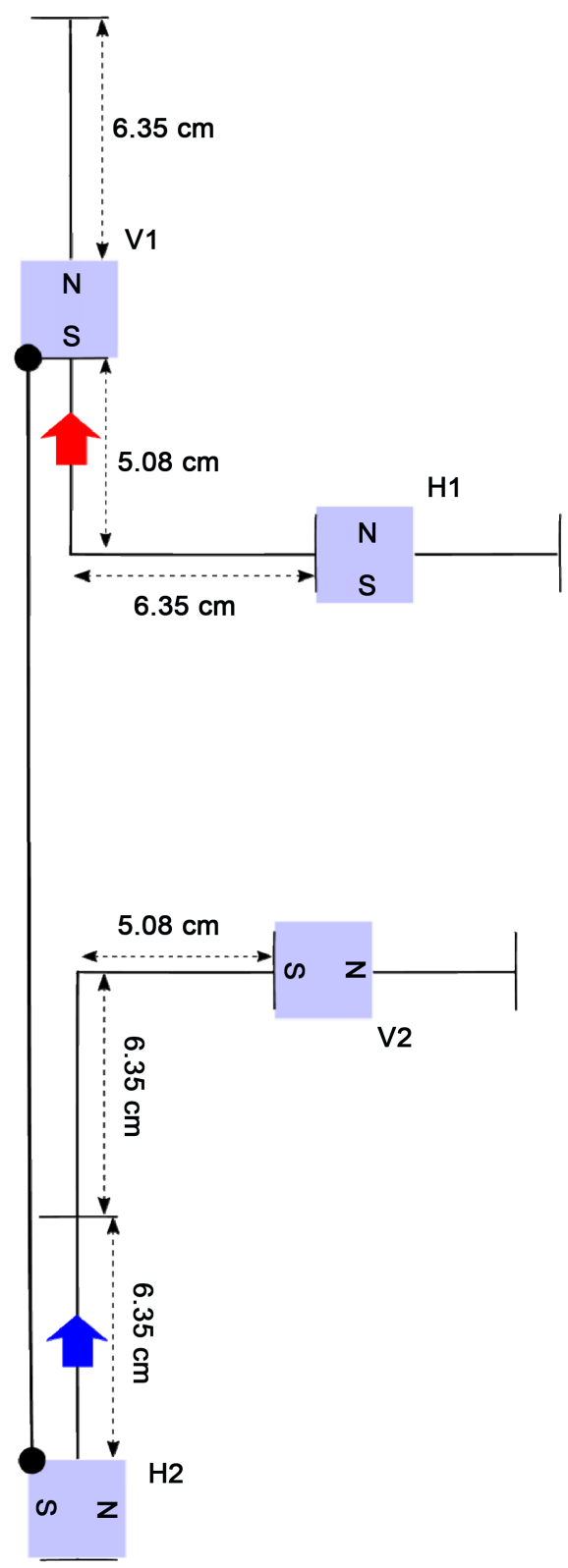

(a)

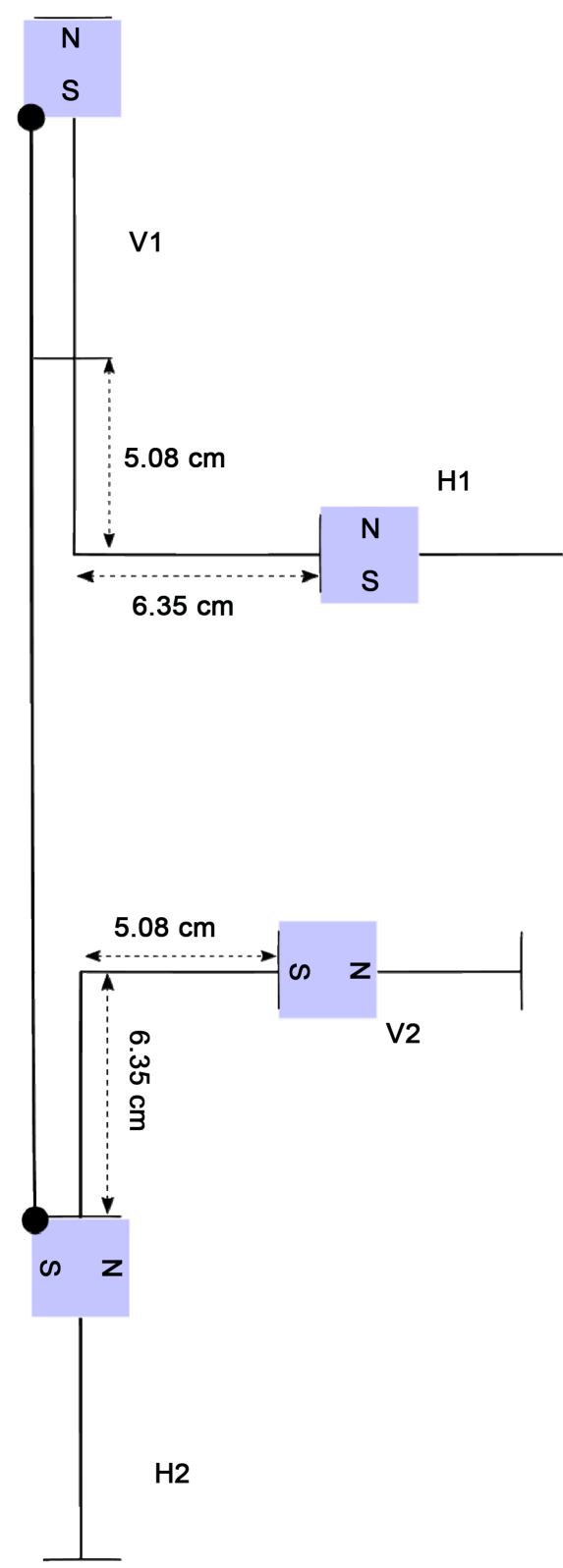

(b)

Figure 13. Travel distances extended by linking pairs of magnets. Magnet V1 is connected to Magnet $\mathrm{H} 2$ of another pair. (a) A repulsive force (red arrow) exists in the polar plane between magnets V1 and $\mathrm{H} 1$. No significant force exists between magnets V2 and $\mathrm{H} 2$. The repulsive force in the polar plane acting on magnet V1 moves magnet V1 away from magnet $\mathrm{H} 1$ and tows magnet $\mathrm{H} 2$ toward magnet $\mathrm{V} 2$. When magnet $\mathrm{H} 2$ moves closer to magnet V2 an attractive force (blue arrow) in the equatorial plane begins to pull magnet $\mathrm{H} 2$ towards magnet $\mathrm{V} 2$. This attractive force will increase in strength as magnet $\mathrm{H} 2$ moves closer to magnet V2 allowing magnet $\mathrm{H} 2$ to tow magnet V1 further away from magnet $\mathrm{H} 1$; (b) Magnets $\mathrm{V} 1$ and $\mathrm{H} 2$ have traveled a further distance that either would have traveled alone. An apparatus that demonstrates motion of the permanent magnets linked together in this manner is shown in Movie S4. 
The amount of mechanical energy that can be extracted from permanent magnets is immense and highly practical especially when compared to fossil fuels and solar (including wind) energy. The amount of energy used to extract, process and distribute fossil fuels along with the amount of fossil fuel used per household in one year alone is immensely greater than the energy and materials needed to provide a household with a lifetime of energy from permanent magnets. Unlike fossil and nuclear fuels, a permanent magnet motor generates electricity without producing pollutants. Permanent magnets can be recycled by restoring the alignment of their dipoles which slowly move out of alignment.

This new energy technology can be implemented rapidly and globally. The mechanical energy available from a permanent magnet machine can be used to generate electricity delivered through our current grid and as a standalone unit providing electricity off the grid. Electricity produced from permanent magnet machines can be used to extract hydrogen from ocean water. With minor modifications, hydrogen can replace petroleum fuels in our current combustion engines. Together, the electricity and hydrogen produced from permanent magnet motors can be used without a major change in our current infrastructure making them highly practical and feasible. Electricity produced from permanent magnet machines can also desalinate ocean water by distillation providing water for drinking and farming in places where fresh water is scarce. Electromagnetic energy derived from permanent magnets in the form of mechanical energy is a clean, safe, abundant and recyclable source of atomic energy that is direly needed. This new technology should be implemented rapidly and globally.

\section{REFERENCES}

1. Uhlenbeck, G.E. and Goudsmit, S. (1926) Spinning Electrons and the Structure of Spectra. Nature, 117, 264-265. https://doi.org/10.1038/117264a0

2. Foley, H. and Kusch, P. (1948) On the Intrinsic Moment of the Electron. Physical Review, 73, 412-412. https://doi.org/10.1103/PhysRev.73.412

3. Feynman, R.P., Leigton, R.B. and Sands, M. (1964) The Feynman Lectures on Physics. Addison-Wesley Publishing Company, Boston, 2, 7. https://doi.org/10.1063/1.3051743

4. Peskin, M.E. and Schroeder, D.V. (1995) An Introduction to Quantum Field Theory. Westview Press, New York.

5. Penrose, R. (1989) The Emperor's New Mind: Concerning Computers, Minds, and the Laws of Physics. Oxford University Press, Oxford.

6. Eric, W.L. (1963) Magnetism: An Introductory Survey. Courier Dover Publications, New York. 


\section{APPENDIX}

The movies S1-S5 reported in this paper are available at http://www.kedronenergy.com.

Movie S1-Equatorial attract. An apparatus demonstrates horizontal motion produced by the resultant attraction in the equatorial plane between two permanent magnets with their opposite poles facing one another.

Movie S2-Polar repel. An apparatus demonstrates vertical motion produced by the resultant repulsion in the polar plane between two permanent magnets with their opposite poles facing one another.

Movie S3-Attract-repel sequence. An apparatus demonstrates in sequence the equatorial attraction and polar repulsion shown in Movies S1 and S2 respectively.

Movie S4-An apparatus demonstrates magnet pairs linked together out of phase to illustrate increased travel distance for both horizontal and vertical motion.

Movie S5-Continuous motion from 4 pairs. An animation demonstrates continued motion by linking four pairs of magnets.

\section{Submit or recommend next manuscript to SCIRP and we will provide best service for you:}

Accepting pre-submission inquiries through Email, Facebook, LinkedIn, Twitter, etc.

A wide selection of journals (inclusive of 9 subjects, more than 200 journals)

Providing 24-hour high-quality service

User-friendly online submission system

Fair and swift peer-review system

Efficient typesetting and proofreading procedure

Display of the result of downloads and visits, as well as the number of cited articles

Maximum dissemination of your research work

Submit your manuscript at: http://papersubmission.scirp.org/

Or contact ns@scirp.org 